\title{
The relationship between naming and semantic knowledge for different categories in dementia of Alzheimer's type
}

\author{
MATTHEW A. LAMBON RALPH, ${ }^{*}$ KARALYN PATTERSON $\dagger$ and JOHN R. HODGES†† \\ *Department of Psychology, University of York, York, U.K.; †MRC Applied Psychology Unit, Cambridge, U.K.: \\ †University Neurology Unit, University of Cambridge. Cambridge CB2 2QQ. U.K.
}

(Received 9 October 1996; accepted 10 March 1997)

\begin{abstract}
We studied the relationship between naming and semantic memory in a group of 10 patients with dementia of Alzheimer's type. In an extension to a previous cross-sectional study (Hodges, J. R. et al. Brain and Language, 1996, 54, 302 325), this relationship was investigated at two longitudinal points within each patient's cognitive decline. Two types of naming performance were compared: items that each patient named correctly at the first stage but failed to name at the second stage, as contrasted with items named correctly at both stages (thereby providing a control for cognitive decline in general). Semantic knowledge of the concepts represented by the pictures in the naming test was investigated at each stage using definitions to the spoken object name, scored particularly for the number of sensory and associative/functional features provided by the patient.

At stage 2, an analysis of the definitions for named $\rightarrow$ unnamed items as contrasted with named $\rightarrow$ named objects revealed a significant loss of both sensory and associative information. A comparison between natural kinds (animals and birds) and artefacts (household objects, vehicles, etc.), however, demonstrated a striking interaction between category and type of information contained in the definitions. Specifically, stage 2 definitions of artefacts in the named $\rightarrow$ unnamed set showed a disproportionate loss of associative/functional information, while definitions of animal names that patients failed to produce in response to the pictures were notably lacking in sensory features. This pattern supports the notion that successful naming relies on a subset of critical semantic features which vary somewhat across different categories of semantic knowledge. We suggest that these findings are best encompassed by a conception of semantic organization, Weighted Overlappingly Organized Features (WOOF), in which (i) knowledge about all objects is represented by a central, distributed network of features activated by both words and pictures, but (ii) natural kinds and artefacts are differentially weighted in favour of those features that are involved in learning about and experiencing different kinds of objects. if 1997 Elsevier Science Ltd.
\end{abstract}

Key Words: Alzheimer's disease: naming; semantic memory: category specificity.

\section{Introduction}

Semantic memory is the basis on which we give meaning to our interaction with the world. It represents our conceptual knowledge of objects and facts, and the meanings of words [24, 25]. The internal structure of semantic knowledge is still subject to much debate, with particular controversy in at least two respects: are there separate domains of representation either for different types of semantic category (particularly natural kinds vs artefacts) or for different modalities of access [objects (or pictures of them) v's words referring to the objects]? Analyses

\Address for correspondence: University Neurology Unit, Addenbrooke's Hospital. Hills Road, Cambridge CB2 2QQ, U.K.: tel.: 01223 217697; fax: 01223336941 ; e-mail: jrh24 (a cus. cam.ac.uk. of patients with selective disorders of semantic memory resulting from brain injury or disease have played a central role in this debate.

We shall divide conceptions of semantic organization into three types: category- and modality-specific, amodal and hybrid hypotheses. Warrington and McCarthy [2628] have proposed that semantic memory systems are fractionated both by category and by modality. They argue that, in principle, there should be, and in fact there are, patients with disorders represented by each of the four 'quadrants' of the two-by-two (category-bymodality) array. For example, patient TOB [15] was impaired in producing information about living things in response to their spoken names, with significantly superior performance in the other three conditions of the quadrant (living things presented in pictorial format, and artefacts denoted by either picture or word). 
A contrasting position proposes an amodal, unitary semantic system (e.g., [2, 5, 10, 19]). This view emphasizes that semantic information, assumed to be represented in one central system, is retrieved and expressed via different input and output processes. Modality-specific effects such as those reported by Warrington and McCarthy are attributed either to a selective disorder of a specific input system or, in the case of an advantage for pictures over words, to the fact that a picture offers more directly inferable information about the nature of the object portrayed than its verbal label can provide.

Finally, one hybrid hypothesis has been described by Chertkow et al. $[3,4]$. In this proposal, semantic memory is split into 'identification semantics' and 'associative semantics'. The former refers to the subset of conceptual knowledge that is necessary for distinguishing, and subsequently naming, individual referents. Associative semantics represents the remaining knowledge about each item which is not required for identification (e.g., encyclopaedic facts). For real objects or their pictures, exemplars must activate identification semantics before achieving access to associative knowledge; words, on the other hand, are thought to allow direct access to associative information. Pictorial stimuli have preferential access to identification semantics in comparison to verbal material.

One line of evidence germane to these models, from patients with semantic deterioration, is an analysis of the relationship between performance in naming visually presented objects and adequacy of knowledge produced in response to the verbal labels of these objects. We shall refer to this as the relationship between naming and knowing. Modality-specific frameworks do not predict any necessary relationship between these two abilities. The availability of knowledge in verbal semantics (as revealed in definition performance) is unrelated to the degree of loss/preservation of corresponding attributes in object semantics (accessed by pictures). For example, the feature striped for the concept of a tiger may be provided in a verbal definition (and thus is present in the verbal semantic system), but may be lost/unavailable for naming (which requires intact object semantics), or vice versa. In contrast, the unitary alternatives predict that anomia should be correlated with degraded semantic knowledge as accessed by words as well as pictures. At least in the current formulations of unitary semantic models, this view probably also expects naming performance to relate to the general amount of degradation but not to any specific subset of attributes (unless accompanied by disruption to a specific input process, e.g., the structural description system [21]). Returning to the example given above, if the feature striped cannot be produced by the patient in a definition it is unlikely that it will be activated when the patient attempts to name the picture of a tiger, because both tasks draw on the same set of semantic attributes. The hybrid scheme of Chertkow et al. predicts that the ability to name an object should relate only to knowledge, as activated by the object's verbal label, of those semantic features required for visual identification (identification semantics) and not to associative features. If this theory is correct then anomia will be correlated with a subset of critical features (perhaps like TIGER: striped, which differentiates this exemplar from other large cats) while naming performance will not be related to the availability of any associative features (e.g., TIGER: carnivore).

One form of brain disease which disrupts semantic knowledge is dementia of the Alzheimer's type (DAT). In a previous cross-sectional analysis of the relationship between naming and knowing in a group of DAT patients, Hodges et al. [13] obtained a significant relationship between the patients' success in naming a set of line drawings of common objects and the amount and type of semantic information generated on a definition task in response to verbal labels for these objects. In the definitions test, the unnamed items (i) yielded relatively less total information, (ii) were less likely to be described to a level that conveyed the 'core' concept of the object, and (iii) of particular interest to this discussion, tended to lack specific physical or sensory, rather than associative, features. This finding of a close relationship between naming and knowing seems more compatible with unitary, amodal proposals than with the assumption that pictures and words access separate pools of conceptual knowledge. On the other hand, these results also support the notion that naming is especially reliant on knowledge of sensory features, a relationship not specifically predicted by most descriptions of a unitary system.

We propose here a slightly different conception of semantic memory which seems consonant with both of these two findings. We shall refer to this conception as Weighted Overlappingly Organized Features, or WOOF for short. In this framework, all concepts are represented in a central, amodal network of semantic features accessed both by seeing an object and by hearing or reading its name; that is the $O O F$ part of the acronym. The $W$ refers to the idea that individual objects will have differential weightings on the types of features that form a 'core' concept of the object [13]. If a sufficient proportion of the features central, say, to the concept of a giraffe cannot be activated, then of course the patient will fail to produce these features in response to the request "Please tell me everything that you know about a giraffe". According to WOOF, however, the patient should also have difficulty in identifying, from the picture of a giraffe, the features critical for differentiating it from other similar objects (e.g., an elephant, rhinoceros or zebra) and thus should fail to name it as 'giraffe'.

It seems likely to us that the organization of networks of knowledge must be determined in large part by the characteristics of the experiences through which the knowledge is acquired. A key aspect of WOOF is therefore that the set of features critically defining an object will vary in a principled way across exemplars drawn from different categories. For natural kinds such as animals, birds, fruit and vegetables, there will be a differ- 
ential weighting in favour of visual/sensory features. This is because, in learning about natural-kind exemplars, we distinguish between them primarily on the basis of their shapes, of how they move (in the case of animals), of their colours and tastes (in the case of fruit), etc. By contrast. there are other categories (typically relating to man-made objects or artefacts) in which the knowledge that differentiates between exemplars is based less on sensory features and more on aspects of the object's function, typical location, etc. In this respect, WOOF derives from the ideas of other researchers who have been prominent in the debate about category-specific semantic disorders $[1,6,9,20,28]$. We shall repeat Warrington and Shallice's examples ([28], p. 849) to illustrate. To distinguish between chalk, crayon and pencil, one needs to know about their 'subtly different functions', while in differentiating amongst lion. tiger and leopard, "sensory attributes provide the definitive characteristics'.

A particular selection of attributes may thus be critical for one set of concepts, while remaining effectively redundant for another. Emphasizing the importance of a subset of features does not, however, necessitate a separate system for them. The theoretical arguments and results of a computational model by Farah and McClelland [6] have been especially influential in supporting this claim. Very different kinds of concepts may be represented by Overlappingly Organized Features in a single network, but still rely on different subsets of critical attributes for identification and subsequent naming. To borrow the term of Hillis et al. [10], such features have varying 'discriminative value' within a single semantic network. The idea of one semantic system has certain advantages: for example, it circumvents vexing questions about whether features must be duplicated across separate systems specialized for different kinds of concepts and/or different modalities of access.

A simple graphical representation of WOOF is shown in Fig. 1. The diagram indicates heavier weighting on features in the sensory (visual) domain than on associative features for natural kinds: in contrast, but as a matter of degree rather than any sharp qualitative distinction, representations for artefacts have greater weighting on features concerned with the way in which we interact with such objects, in particular why and how we use them. This pattern results from the assumptions (i) that sensory and associative semantic features are represented in the same distributed network, but (ii) that these different aspects of central conceptual knowledge are asymmetrically linked to different processing regions in the brain. because (iii) information about different classes of objects is derived, at least in part, from different input (sensory) processing systems, both in the initial learning of an infant and in the continuing experience of a child or adult. Furthermore, as indicated in Fig. 1. WOOF assumes that objects (or pictures) and words activate the same distributed network of semantic features.

The present study was designed to replicate and extend the findings of Hodges et al. [13], using a longitudinal rather than cross-sectional approach. Ten DAT patients were selected who had shown a significant decrease in object-naming performance during their cognitive decline. For each patient, pairs of items were selected such that one item had moved from named to unnamed status (hereafter $\mathrm{N} \rightarrow \mathrm{U}$ ) between stages 1 and 2 of the study, whilst the other remained named throughout $(\mathrm{N} \rightarrow$ $\mathrm{N})$. For each pair, the patient had also provided a verbal definition of each target item in response to the spoken object name at both stages. This within-subject and indeed within-item approach was designed to address the following points:

1. Can we confirm the previous finding of a reliable itemspecific relationship between object-naming accuracy and adequacy irichness of verbal information?

2. Is naming success significantly correlated with a particular subset of semantic features produced in the definitions task?

3. If so. does this critical subset of features vary for exemplars drawn from different categories"

4. Is there evidence for disproportionate loss of associative information in DAT (as proposed by Chertkow et al. [4])?

5. Does success in object naming correlate with knowledge of superordinate category, as measured by the likelihood that a patient's definition mentions what general kind of thing the target is? Hodges et al. [13] failed to find such a relationship.

6. Does the amount of intrusive (incorrect) information included in the patients' definitions increase as a function of cognitive decline. and does this relate to naming accuracy"?

\section{Methods}

Ten DAT patients were selected from a group of approximately 50 patients who were recruited into a longitudinal study of memory and language in DAT. The diagnosis of DAT was made according to the criteria developed by the National Institute of Neurology and Communicative Disorders and Stroke (NINCDS) and the Alzheimer's Disease and Related Disorders Association (ADRDA) [16]. All patients presented with progressive cognitive impairment, predominantly affecting memory, with a history of $1-4$ years. Since 199|-1992, all patients have undergone longitudinal assessment at 6-month intervals for at least 2 years and, in many cases, for as long as $3-4$ years. In all cases there has been a decline in performance affecting at least two areas of cognition. Patients with a past history of known or suspected transient cerebral ischaemic event or stroke, alcoholism, head injury or major medical illness were excluded, as were those with major depression.

The 10 patients selected for this study had all shown a significant decline in their naming performance over time as assessed by a 48-item test of naming to confrontation [12], For each patient, two stages of testing (from the longitudinal assessments) were selected which maximized both the decline in naming performance and the time interval between the two stages whilst ensuring that, at the second stage, the patient had been able to perform the definitions task. Demographic details, 


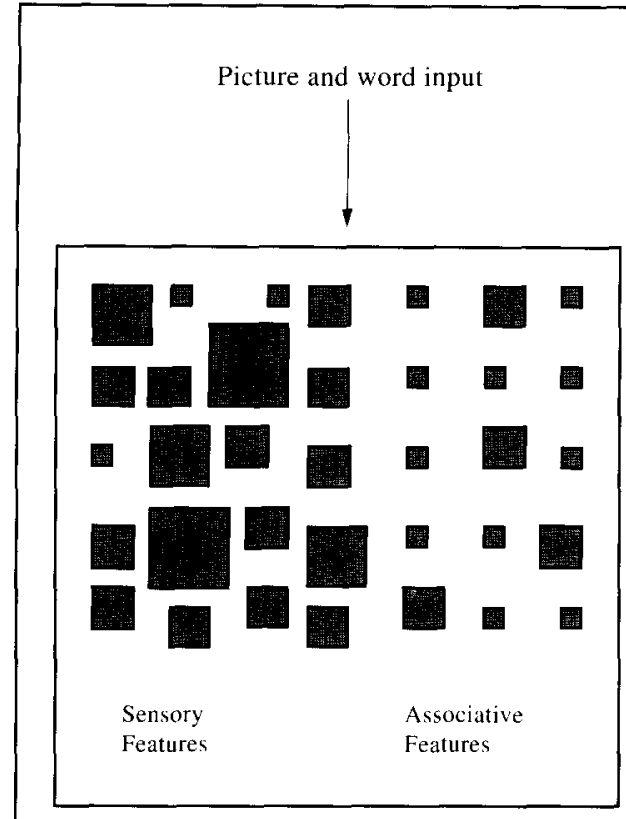

(a) Weighting of semantic features for concepts relating to natural kinds.

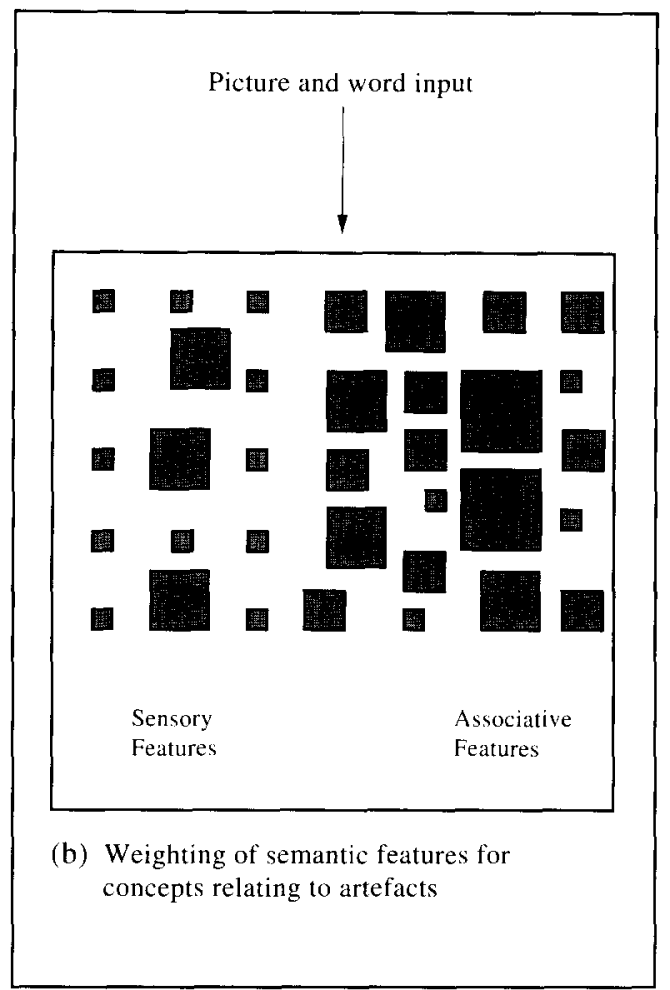

Fig. 1. A simplified depiction of the Weighted Overlappingly Organized Features (WOOF) conception of semantic memory. Features with relatively greater weighting are represented by larger squares.

results of neuropsychological testing at stages 1 and 2, and the number of months intervening between stages 1 and 2 are all given in Table 1. As can be seen, there was considerable longitudinal decline in cognitive performance across the range of tasks. Between stages 1 and 2 , all patients showed significant decrements in naming accuracy on the 48-item test $(t=11.2$, $P<0.001$ ), in general cognitive ability as assessed by the MiniMental State Examination $(t=6.1, P<0.001)$ and in associative semantic knowledge as measured by the three-picture version of the Pyramids and Palm Trees test [14] $(t=4.3$, $P=0.004)$.

\section{Item selection}

At each round of testing, all patients had been asked to name a set of 48 line drawings, half representing animals and half artefacts (from the Snodgrass and Vanderwart corpus [22]), and also to define a subset of these items from the spoken label. The exact method and instructions for the definitions can be found in Hodges et al. [13]. Briefly, the patients were instructed to describe each item as if the listener were unfamiliar with the object; they were given $1 \mathrm{~min}$ to provide as much information as possible for each concept. These definitions were preceded by two practice items (cow and banjo) for which the patient was given feedback and examples of the sort of information they might have provided.

Pairs of items were selected from each patient's corpus for which a definition and a naming response had been given at both stages 1 and 2 . One of the pair was an item which the patient had correctly named at stage 1 but had failed at stage 2 $(\mathrm{N} \rightarrow \mathrm{U}$ items). The other item was named on both occasions by the patient $(\mathrm{N} \rightarrow \mathrm{N})$. A total of 42 pairs of $\mathrm{N} \rightarrow \mathrm{U}$ and $\mathrm{N} \rightarrow$

Table 1. Background neuropsychology results and demographic information

\begin{tabular}{|c|c|c|c|c|c|c|c|c|c|c|}
\hline Patient & Age & $\begin{array}{l}\text { Years of } \\
\text { education }\end{array}$ & $\begin{array}{l}\text { Time } \\
\text { between } \\
\text { stages in } \\
\text { months }\end{array}$ & $\begin{array}{l}\text { Naming at } \\
\text { stage l } \\
(\max .=48)\end{array}$ & $\begin{array}{l}\text { Naming at } \\
\text { stage } 2 \\
(\max .=48)\end{array}$ & $\begin{array}{c}\text { MMSE at } \\
\text { stage } 1 \\
(\max .=30)\end{array}$ & $\begin{array}{c}\text { MMSE at } \\
\text { stage } 2 \\
(\max .=30)\end{array}$ & $\begin{array}{c}\text { PPT at } \\
\text { stage } 1 \\
(\max =52)\end{array}$ & $\begin{array}{c}\text { PPT at } \\
\text { stage } 2 \\
(\max .=52)\end{array}$ & $\begin{array}{l}\text { No. of } \\
\text { item } \\
\text { pairs } \\
\text { selected }\end{array}$ \\
\hline $\mathrm{RB}$ & 78 & 11 & 30 & 40 & 26 & 27 & 18 & 49 & 39 & 6 \\
\hline$A B$ & 73 & 9 & 6 & 42 & 34 & 17 & 7 & 44 & 33 & 3 \\
\hline MB & 61 & 9 & 18 & 32 & 19 & 13 & 8 & - & 31 & 4 \\
\hline LD & 66 & 10 & 24 & 32 & 22 & 19 & 13 & 35 & 32 & 4 \\
\hline $\mathrm{DE}$ & 66 & 11 & 6 & 34 & 25 & 15 & 11 & - & - & 3 \\
\hline PG & 71 & 9 & 18 & 45 & 35 & 23 & 18 & 46 & 38 & 6 \\
\hline BL & 67 & 11 & 18 & 46 & 35 & 23 & 16 & 44 & 37 & 6 \\
\hline MR & 72 & 11 & 12 & 15 & 9 & 15 & 13 & 38 & 35 & 1 \\
\hline SW & 73 & 12 & 6 & 39 & 26 & 20 & 19 & 49 & 46 & 4 \\
\hline $\mathrm{SW}$ & 57 & 11 & 18 & 42 & 36 & 18 & 7 & 47 & 29 & 5 \\
\hline Mean & 68.4 & 10.4 & 15.6 & 36.7 & 26.7 & 19.0 & 12.9 & 44.0 & 36.1 & \\
\hline
\end{tabular}

Naming to confrontation (see [12]). MMSE: Mini-Mental State Examination [7]. PPT: Pyramids and Palm Trees test [14]. 
$\mathrm{N}$ items fitted all of the selection criteria; the number selected from each patient is shown in Table $\mathrm{I}$. Where possible, $\mathrm{N} \rightarrow \mathrm{U}$ items were chosen such that naming performance had been consistent: that is, once the patient failed to name that item, he or she never succeeded in naming it at a later test session. Only one of the $42 \mathrm{~N} \rightarrow \mathrm{U}$ items did not conform to this criterion Furthermore. no $\mathrm{N} \rightarrow \mathrm{U}$ items were selected for which the misnaming involved either a phonological or purely visual error: all errors were either semantic or failures to respond. The selected set included 44 animals and 40 artefacts.

Definitions were evaluated on a basis similar to that described in Hodges el $a l$. [13]. Each was scored for the number of specific sensory features (which mainly included visual features, but also some characteristic sounds), for the number of specific associative/functional attributes (e.g., how the object is used, where it is found, etc.), for the presence of a superordinate label or description (e.g., "it's an animal". "that's a thing for making music") and for the number of intrusions (incorrect information). Additionally, the correct sensory, associative: functional and superordinate scores were summed to yield a measure of total amount of correct information provided by each patient for each item at each stage. For the purposes of this analysis. we did not include the categories of general sensory information (e.g.. "it has eyes, legs" etc.) and general associative information (e.g.. "you use it, play it" etc.). As shown in Hodges et al. [13] (Table 2, p. 310), our previous study of the relationship between naming and definitions revealed that both normal controls and the DAT patients produced very little information of this type.

Differences between item group $(\mathrm{N} \rightarrow \mathrm{U}$ vs $\mathrm{N} \rightarrow \mathrm{N})$ and stage ( 1 vs 2) were tested using a series of $2 \times 2$ split-plot analyses of variance (ANOVAs). Where significant interactions occurred, planned comparisons were performed using Student's $t$-tests.

\section{Results}

We report the results below in relation to the specific questions set at the end of the Introduction.

\section{Is there a reliahle relationship between naming accuracy. and adequacyichness of verbal information?}

Figure 2 represents the mean total amount of correct information given in the definitions for $\mathrm{N} \rightarrow \mathrm{U}$ and $\mathrm{N} \rightarrow$ $N$ stimuli, and reveals a disproportionate loss of information in the $\mathrm{N} \rightarrow \mathrm{U}$ set in comparison to $\mathrm{N} \rightarrow \mathrm{N}$ items. The $2 \times 2$ ANOVA revealed a main effect of stage $[F(1,82)=97.7 . P<0.001]$, no main effect of item type $[F(1.82)<1]$ and a significant stage $\times$ item type interaction $[F(1.82)=34.38, \quad P<0.001]$. Planned comparisons revealed a significant difference between stages 1 and 2 for both the $\mathrm{N} \rightarrow \mathrm{U}$ and $\mathrm{N} \rightarrow \mathrm{N}$ sets (paired $t$ tests: $t=9.96, P<0.001$ and $t=3.40, P=0.002$. respectively).

This result corroborates. longitudinally, what Hodges ('t al. [13] demonstrated cross-sectionally. That is, although less definitional information overall was provided by the DAT patients as their disease progressed. there was a significantly greater loss of information for items that had changed status from named to unnamed

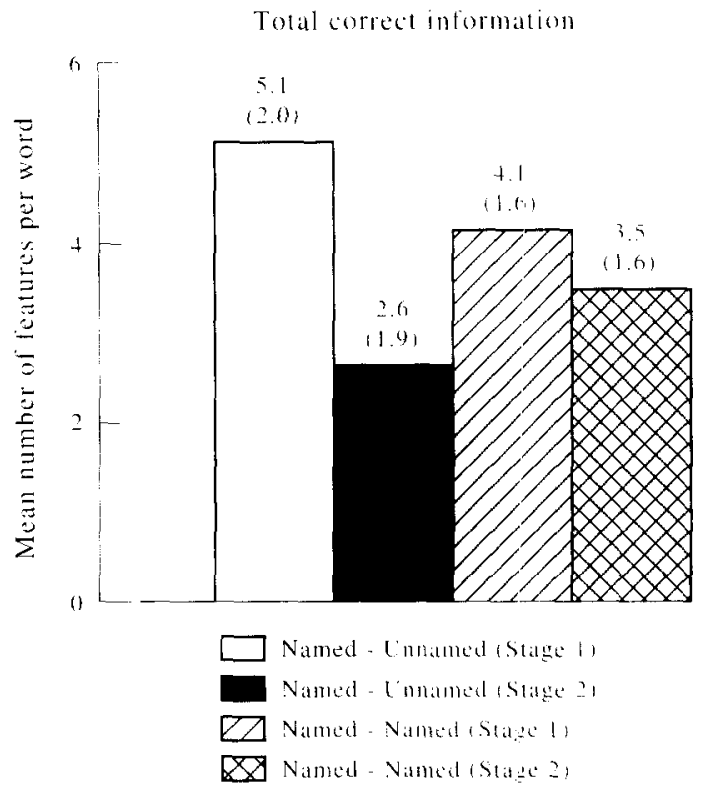

Fig. 2. The mean number of total correct features per word for Named - Unnamed and Named Named items at stages 1 and 2 (slandard deviations in parentheses).

$(\mathrm{N} \rightarrow \mathrm{U})$ in comparison to those items that were correctly named at both stages.

Is naming performance significantly correlated with a particular subset of semantic features produced in the definitions task?

Figures $3 a$ and $b$ reveal that the definitions contained a remarkably similar number of the two major classes of features. That is, the notable reduction in information provided at stage 2 for $\mathrm{N} \rightarrow \mathrm{U}$ items applied to both sensory and associative functional information. The $2 \times 2$ analysis yielded the same pattern for both types of information: significant main effects of stage $[F(1,82)=27.1$. $P<0.001$ and $[F(1,82)=24.0, \quad P<0.001]$. non-significant main effects of item type $[F(1.82)=1.38$. n.s. and $F(1.82)=1.18$. n.s.] and significant interactions $[F(1.82)=14.35, \quad P<0.001$ and $F(1.82)=6.2, \quad P=$ $0.015]$. Planned comparisons for sensory information showed that the difference between stages 1 and 2 was significant for the $\mathrm{N} \rightarrow \mathrm{U}$ items (paired $t$-test: $t=6.23$. $P<0.001$ ), but not for the $\mathrm{N} \rightarrow \mathrm{N}$ items (paired $t$-test: $t=1.03$ n.s.). Similar planned comparisons for associative/functional information yielded a reliable difference between stages 1 and 2 for the $\mathrm{N} \rightarrow \mathrm{U}$ items (paired $t$ test: $t=4.61, P<0.001)$ and a difference of borderline significance for the $\mathrm{N} \rightarrow \mathrm{N}$ items (paired $t$-test: $t=2.01$. $P=0.05)$.

These longitudinal data suggest that failure to name a target picture is correlated with a loss of both sensory and associative functional information. 
(a)

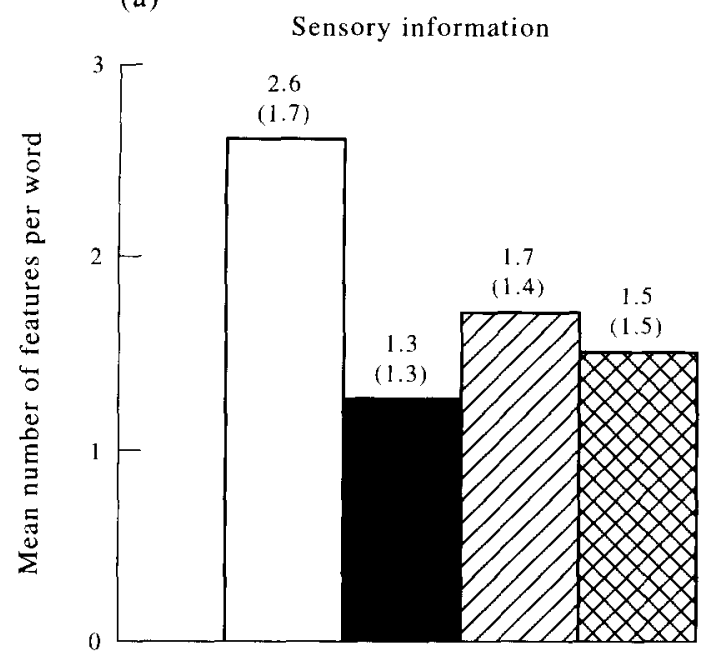

(b)

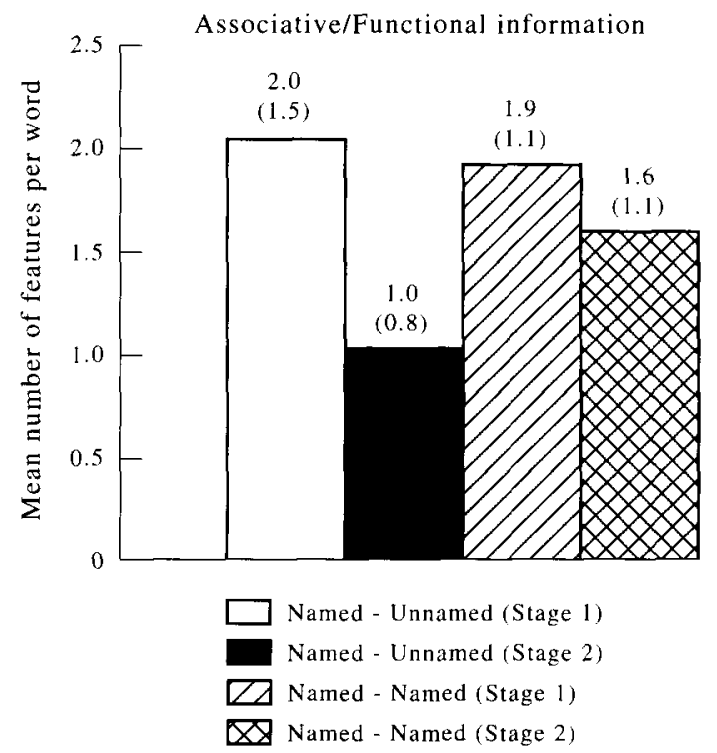

Fig. 3. The mean number of sensory and associative/functional features per word for Named-Unnamed and Named-Named items at stages 1 and 2 (standard deviations in parentheses)

Does naming of exemplars drawn from different categories relate to different subsets of definitional features?

In order to assess the relationship between object category and the nature of semantic features critical for naming, we split the $\mathrm{N} \rightarrow \mathrm{U}$ and $\mathrm{N} \rightarrow \mathrm{N}$ items into natural kinds (animals and birds) and artefacts (musical instruments, vehicles and household objects). The number of sensory and associative/functional features in the definitions to items from these two broad categories are illustrated in Fig. 4, which reveals a striking category effect in the relationship between naming and featural information. For living things, $\mathrm{N} \rightarrow \mathrm{U}$ and $\mathrm{N} \rightarrow \mathrm{N}$ items are differentiated in terms of sensory features, with a marked decrease in this type of information between stages 1 and 2 for $\mathrm{N} \rightarrow \mathrm{U}$ but no change for $\mathrm{N} \rightarrow \mathrm{N}$ (Fig. 4a). By contrast, $\mathrm{N} \rightarrow \mathrm{U}$ and $\mathrm{N} \rightarrow \mathrm{N}$ natural kinds are not differentiated in terms of functional features, which decreased by only a small amount and about equally for the two naming groups (Fig. 4b). Artefacts showed the reverse pattern. Here, it was the number of functional features that decreased notably between stages 1 and 2 for $\mathrm{N} \rightarrow \mathrm{U}$ but not for $\mathrm{N} \rightarrow \mathrm{N}$ (Fig. 4d), whilst the number of sensory features declined to a much smaller extent between stages, and about equally for the two sets (Fig. $4 c)$.

This qualitative description is statistically confirmed by the $2 \times 2$ split-plot ANOVAs and planned comparisons. The analysis of sensory information for animate targets (Fig. 4a) and of associative/functional features for man-made objects (Fig. $4 \mathrm{~d}$ ) revealed significant main effects of stage $[F(1,42)=10.75, \quad P=0.002$ and $F(1,38)=17.21, P<0.001]$, non-significant main effects of item type (both $F \mathrm{~s}<1$ ) and significant interactions $[F(1,42)=10.75, \quad P=0.002 \quad$ and $\quad F(1,38)=10.28$, $P=0.003$, respectively]. Planned comparisons for both sets of data showed significant differences between stages 1 and 2 for the $\mathrm{N} \rightarrow \mathrm{U}$ items (paired $t$-tests: $t=5.95$, $P<0.001$ and $t=3.50, P=0.004$ ), but not for the $\mathrm{N} \rightarrow$ $\mathrm{N}$ items (paired $t$-tests: $t=0$, n.s. and $t=1.0$, n.s., respectively). The contrasting pattern for associative/functional information on animate targets (Fig. 4b) and sensory features on man-made objects (Fig. 4c) was confirmed by the ANOVAs: significant main effects of stage $[F(1,42)=11.32, P=0.002$ and $F(1,38)=11.19$, $P=0.002]$, non-significant main effects of item type $[F(1,42)=1.44$, n.s. and $F(1,38)<1]$ and non-significant interactions $[F(1,42)<1$ and $F(1,38)=1.35$, n.s., respectively].

The results demonstrated in Fig. 4 suggest a categoryspecific difference in the critical features needed to name an item. The information critical for the correct naming of natural kinds seems to be biased towards sensory features, while the information critical for artefacts is more weighted toward functional/associative aspects of conceptual knowledge. There was no principled relationship between naming accuracy and associative/functional information for animate targets nor between naming accuracy and sensory information for man-made targets, but rather a small and parallel decline in both types of information, in line with increasing cognitive disruption.

\section{Is there evidence for disproportionate loss of associative information in dementia of Alzheimer's type?}

The mean numbers of sensory and functional attributes provided by the patients per target are shown in Fig. 5 . A $2 \times 2$ repeated-measures ANOVA revealed a significant main effect of stage $[F(1,83)=60.6, P<0.001]$, but no main effect of feature type $[F(1,83)<1]$ or interaction $[F(1,83)<1]$. Thus, contrary to the hypothesis proposed by Chertkow et al. [4], but in keeping with the crosssectional findings of Hodges et al. [13], there was neither a difference between associative and sensory information overall, nor any evidence that the loss of these two classes 
(a)

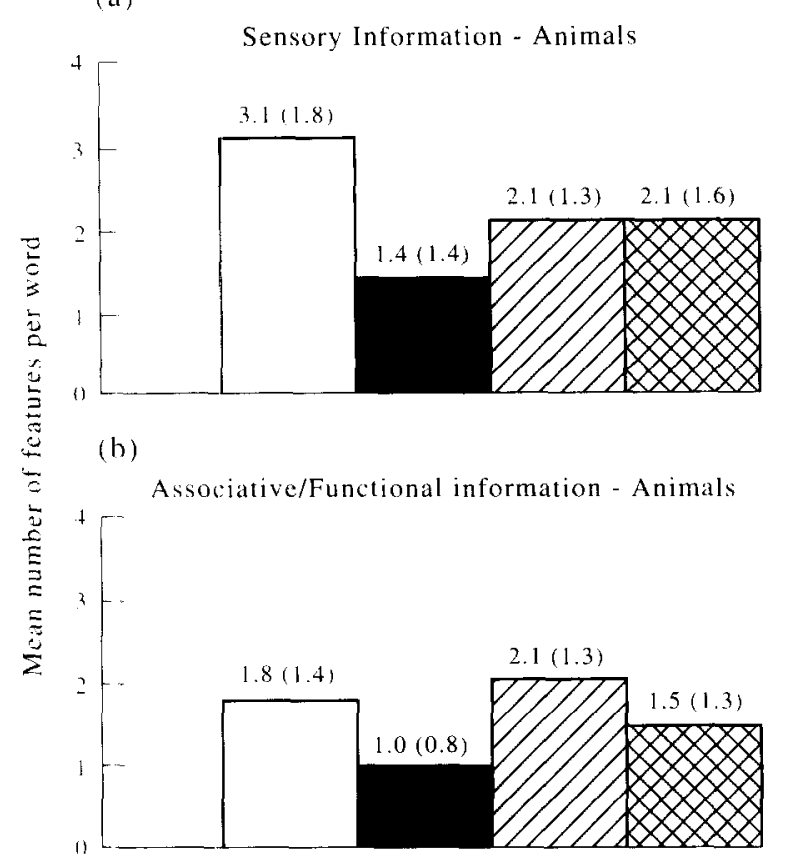

(c)

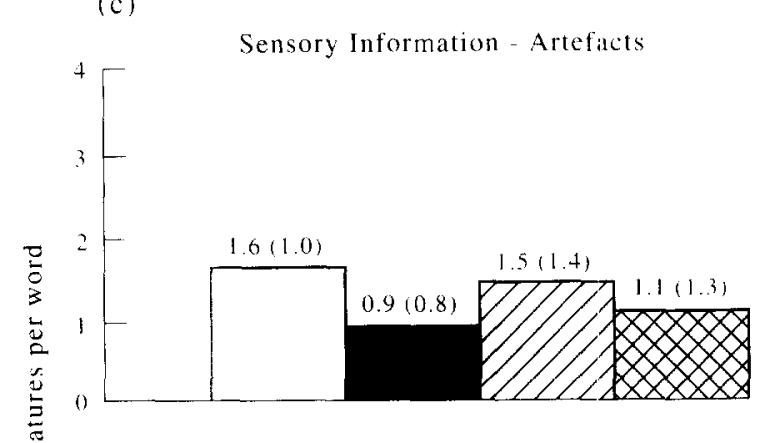

(d) Assotiative/Functional Information - Artefacts

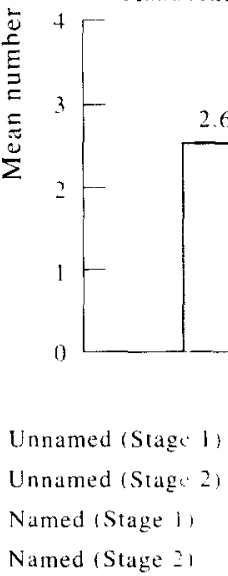

Fig. 4. The mean number of sensory and associative/functional features per word for Named-Unnamed and Named Named items, split for animals and artefacts, at stages 1 and 2 (standard deviations in parentheses).

of feature occurs at differential rates during the course of the patients' decline. As would be expected, the total amount of information that the DAT patients could provide decreased significantly over time, but both types of information declined in parallel.

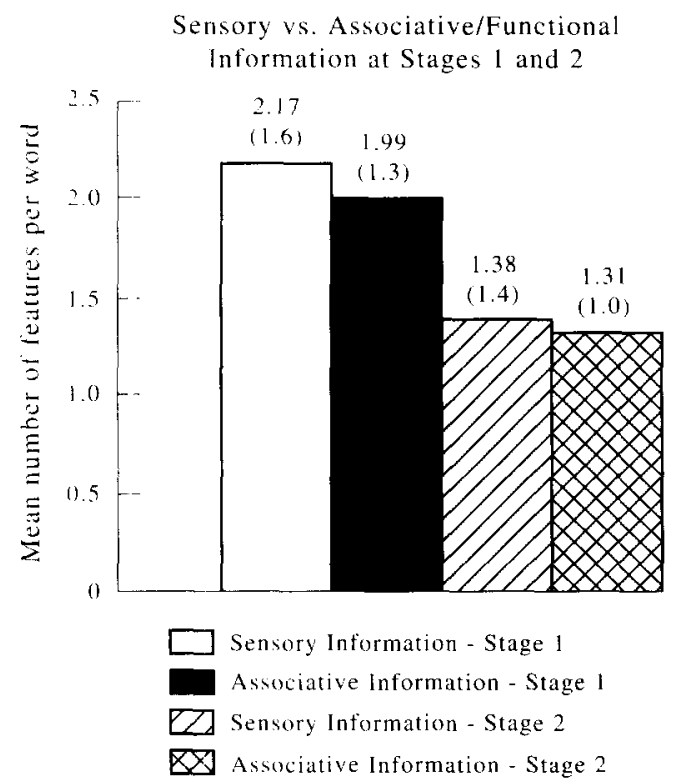

Fig. 5. The mean number of sensory and associative/functional features per word at stages 1 and 2 (standard deviations in parentheses).
Is there a relationship between naming and superordinate knowledge."

The number of superordinate labels or comments provided by the patients for each item type $(\mathrm{N} \rightarrow \mathrm{U}$ vs $\mathrm{N} \rightarrow$ $\mathrm{N})$ and stage are shown in Fig. 6. A $2 \times 2$ ANOVA revealed a reliable effect of stage $[F(1,82)=5.48 . P=$

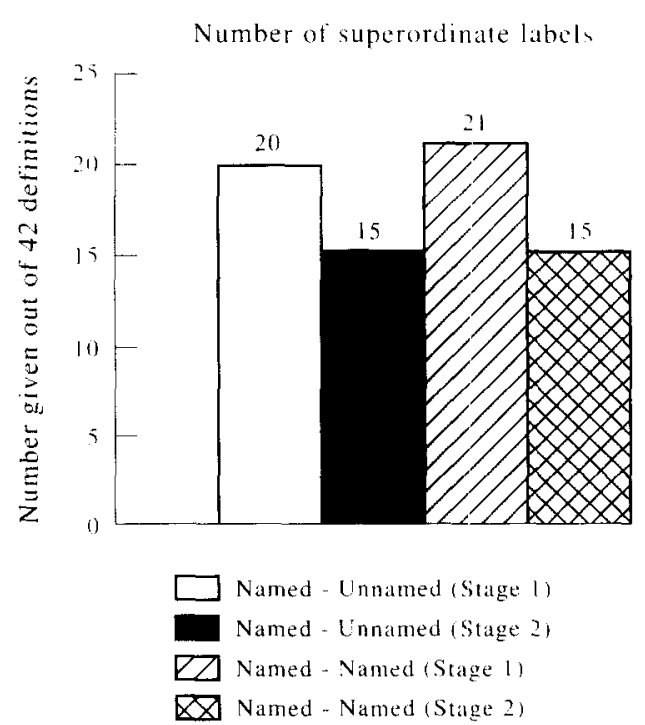

Fig. 6. The total number of superordinate labels given to Named-Unnamed and Named-Named items at stages 1 and 2. 
$0.02]$, but neither a main effect of item type $[F(1,82)<1]$ nor an interaction $[F(1,82)<1]$. As the patients became more impaired during the course of dementia, they were significantly less likely to include a superordinate label within their definitions, but this effect was unrelated to naming success. This result also mirrors the findings of Hodges et al. [13]. Clearly, this measure is based on the spontaneous production of superordinate information by the patients. We believe that the findings would have been similar even if the patients had been asked explicitly to produce superordinate labels, though of course we cannot confirm this a posteriori.

Does the amount of incorrect information in the definitions increase over time, and does this relate to naming accuracy?

The DAT patients in this study produced very little in the way of incorrect information (intrusions). Combining all the definitions across item type and stage, only 38 incorrect features were produced: this is, on average, less than one intrusion for every four definitions. Given these apparent floor effects, it is unsurprising that the ANOVA failed to reveal any significant effects [item type: $F(1,82)<1$; stage: $F(1,82)<1$; interaction: $F(1,82)=$ 2.54 , n.s.]. As very few intrusions were produced, no further statistical analyses were carried out.

From these data it seems that DAT patients do not begin to produce a significant amount of inaccurate information as their disease progresses, nor does this infrequent type of error interact with naming accuracy. A possible relationship may, of course, have been masked by the floor effect; this issue could only be addressed with a larger sample of definitions, or-if patients are still able to cope with the demands of the definitions task - at a later stage in the disease process.

\section{Discussion}

In this longitudinal study, DAT patients' naming accuracy in response to line drawings of common objects was related to the amount of correct semantic information given in response to the spoken names of the objects. In particular, we observed a depletion of both sensory and associative/functional information in the definitions of target items that had changed status from named to unnamed (in comparison to items that continued to be correctly named) during the course of the patients' cognitive decline. Perhaps the most theoretically important finding is the demonstration that naming success appears to relate to a critical subset of semantic features, and that the nature of these critical features depends on the conceptual category of the target item. For artefacts, associative/functional information seems to be more central, while for natural kinds, sensory features have greater prominence. This weighting towards sensory features for animate objects and functional features for inanimate objects is, of course, an idea proposed in many recent discussions of the organization of semantic memory $[1$, $6,9,20,28]$.

This conclusion is based to some degree on logical inference. That is, the analysis does not specifically establish either (i) that individual features provided for $\mathrm{N} \rightarrow$ $\mathrm{U}$ items at stage 1 were missing from stage 2, or (ii) that the lost features are those that are critical for naming. We claim, however, that both of these facts essentially must be true. For the former point, there may indeed have been the occasional instance of a feature (e.g., TIGER: striped) that a patient did not provide at stage 1 but did at stage 2 . On the whole, however, since the analysis is both within patient and within item, and as the number of features declined significantly, the features provided at stage 2 must be very nearly a genuine subset of those from stage 1 . Were this not true, it would seem to imply that the patients were accessing a different knowledge base at stage 2, which seems implausible. For the second point, we have previously documented [13] the fact that, when asked to define these objects/concepts, neither normal controls nor patients with DAT respond with any notable number of very general features that do not distinguish between different exemplars of the target item's category (e.g., "you use it", "it has legs", etc.). Given that the great majority of features produced do reflect knowledge specific to the target item, it must be these that decline in parallel with naming.

In the Introduction, we outlined three somewhat different conceptions of semantic memory discussed in the literature: (i) proposals which assume that pictures and words access separately represented pools of knowledge (e.g., [26, 27]); (ii) those which assume a single system of semantic knowledge, accessed by both pictures and words via differing input processes [2, 6, 19]; and (iii) a proposal distingushing between two systems differentiated not principally by stimulus modality but rather by features critical for identification and naming (identification semantics), in contrast to more associative aspects of object knowledge. We also described our own formulation, Weighted Overlappingly Organized Features (WOOF), in which pictures and words activate features in a single, distributed network that are weighted towards those with a high 'discriminative value' for the object in question. Of the three previous proposals, WOOF is most similar to the second one. How do the present results fit with these various proposals?

First of all, the confirmation of a reliable relationship between naming (in response to pictures) and knowing (in response to words) is consistent with any theory in which pictures and words activate a common semantic network. Secondly, at least on the face of it, the finding of parallel loss of sensory and associative/functional information for unnamed items seems to support a common-network view like the Organized Unitary Content Hypothesis [2] which, at least in its current formulation, does not emphasize the role of any specific subset of features for correct identification. This rather bland 
result, however, conceals the more striking finding that naming accuracy relates to a specific subset of features which themselves vary according to the category of the target items in question. This result fits well with conceptions like WOOF and its relatives (e.g., [6]), in which sensory information is considered more critical for the identification of some categories (particularly natural kinds) and functional features for others (predominantly man-made objects).

As previous authors have noted $[1,6,9,10,20,28]$, it seems likely that features will have differing discriminative values for different types of objects, owing to the varying nature of our interaction with them. If one then considers the pattern of semantic deterioration in the DAT patients studied here, i.e. that all types of features are becoming degraded, the category-specific nature of the relationship between critical features and target pictures is a predictable consequence of the weighting on sensory features for animate targets and on associative/functional features for man-made items. It is important to reiterate that the patients also showed reduced production of sensory information for artefacts and of associative functional information for animate targets. This is to be expected on the assumption that conceptual knowledge is embodied in one distributed network rather than in separate systems. but this kind of degradation had no measurable impact on naming accuracy because these particular combinations of attributes and categories have lower weightings.

In closing. we should mention again the lack of any apparent relationship in our data between naming accuracy and the production of some sort of superordinate label or comment in the corresponding definitions, echoing similar findings by Funnell [8] and Hodges et al. [11. 13]. To the extent that one can interpret negative results, this absent relationship supports a characterization of semantic degradation in which it is the progressive blurring or loss of distinctive features that leads to anomia. Given that a very limited number of features might support knowledge of an object's superordinate class, both relative preservation of performance in tasks requiring only superordinate classification and the lack of predictability between naming success and superordinate information are sensible outcomes (also see $[17,18,23]$ ).

Acknouledgements-We are grateful to Kate Dawson. Kim Graham, Naida Graham, Elaine Hoffner, Sarah Ross and Lindsay Stuart-Green for assistance with various aspects of testing and datil analysis in this study. The research was supported by grants from the Engineering and Physical Sciences Research Council and the Experimental Psychology Society (U.K.) to M.A.L.R. and from the Medical Research Council (U.K.) to J.R.H. K.P.'s contribution was funded in part by a grant from the National Institutes of Mental Health (U.S.A.) to J. L. McClelland.

\section{References}

1. Allport. D. A., Distributed memory, modular subsystems and dysphasia. In Current Perspectives in Drsphasia. ed. S. K. Newman and R. Epstein. Churchill Livingstone, Edinburgh, 1985, pp. 32 60.

2. Caramazıa. A.. Hillis, A. E., Rapp, B. C. and Romani. C.. The multiple semantics hypothesis: multiple confusions. Cognitive Neuropsyology 7, 161 189. 1990.

3. Chertkow. H. and Bub, D., Semantic memory loss in dementia of Alzheimer's type. Brain 113, $397-417$. 1990 .

4. Chertkow, H.. Bub, D. and Caplan. D., Constraining theories of semantic memory processing: evidence from dementia. Cognitive Neuropsycholog! 9, 327 365. 1992.

5. Ellis, A. W. and Young. A. W.. Human Cognitice Neuropsichologl. Lawrence Erlbaum, Hove, U.K. 1988.

6. Farah. M. J. and MeClelland. J. L. A computational model of semantic memory impairment: modality specificity and emergent category specificity. Journal of Experimental Psychology: General 120, $339-357$. 1991.

7. Folstein. M. F., Folstein. S. E. and McHugh, P. R.. "Mini-mental state". A practical method for grading the mental state of patients for the clinician. Joumal of Psuchiatric Research 12, 189 198. 1975.

8. Funnell. E.. Objects and properties: a study of the breakdown of semantic memory. Memory 3, 497 518.1995.

9. Gainotti. G. and Silveri. M. C., Cognitive and anatomical locus of lesion in a patient with a categoryspecific semantic impaiment for living beings. (ognitive Nitropstcholog1 13, 357390.1996.

10. Hillis, A. E., Rapp, B. and Caramarza, A., Constraining claims about theories of semantic memory: more on unitary versus multiple semantics. Cognitice Neuropurchology 12, 175 186. 1995.

11. Hodges. I. R.. Graham. N. and Patterson. K.. Charting the progression in semantic dementia: implications for the organisation of semantic memort. Memorl 3, 463 495. 1995.

12. Hodges. J. R. and Patterson. K.. Is semantic memory consistently impaired carly in the course of Alzheimer' disease? Neuroanatomical and diagnostic implications. Neuropsichologia 33, $441459,1995$.

13. Hodges. J. R. Patterson. K. Graham, N. and Dawson. K.. Naming and knowing in dementia of Alzheimer"s type. Brain and Languacse 54, 302325. 1996.

14. Howard. D. and Patterson. K.. The Promids and Palm Trees Test. Thames Valley Tesi Company. Bury Si Edmunds. 1992.

15. McCarthy, R. A. and Warrington. E. K.. Evidence for modality-specific meaning systems in the brain. Nature 334, 428-430, 1988.

16. McKhann. G., Drachman. D.. Folstein, M.. Katzman. R., Price. D. and Stradlan. E. M.. Clinical diagnosis of Alzheimer's disease. Vemolon. 34, 939 944, $19 \times 4$.

17. Patterson. K. and Hodges. J. R.. Disorders of semantic memory. In Handhook of Memory Disorders, ed. A. D. Baddeley. B. A. Wilson and F. Watts. Wiley, Chichester, 1995. pp. 167.186.

18. Rapp. B. and Caramazra. A. On the distinction 
between deficits of access and deficits of storage: a question of theory. Cognitive Neuropsychology $\mathbf{1 0}$, 113-141, 1993.

19. Riddoch, M. J., Humphreys, G. W., Coltheart, M. and Funnell, E., Semantic systems or system? Neuropsychological evidence re-examined. Cognitive Neuropsychology 5, 3-25, 1988.

20. Saffran, E. M. and Schwartz, M. F., Of cabbages and things: semantic memory from a neuropsychological perspective a tutorial review. In Attention and Performance $X V$, ed. C. Umilta and M. Moscovitch. Lawrence Erlbaum, Hove, U.K., 1994, pp. 507-535.

21. Sartori, G., Job, R., Miozzo, M., Zago, S. and Marchiori, G., Category-specific form-knowledge deficit in a patient with herpes-simplex encephalitis. Journal of Clinical and Experimental Neuropsychology 15, $280-299,1993$.

22. Snodgrass, J. G. and Vanderwart, M., A standardised set of 260 pictures: norms for name agreement, image agreement, familiarity, and visual complexity.
Journal of Human Experimental Psychology: Learning and Memory 6, 174-215, 1980.

23. Tippett, L. J., McAuliffe, S. and Farah, M. J., Preservation of categorical knowledge in Alzheimer's disease: a computational account. Memory 3, 519-533, 1995.

24. Tulving, E., Episodic and semantic memory. In Organisation of Memory, ed. E. Tulving and W. Donaldson. Academic Press, New York, 1972, pp. 382-403.

25. Tulving, E., Elements of Episodic Memory. Clarendon Press, Oxford, 1983.

26. Warrington, E. K. and McCarthy, R. A., Categories of knowledge: further fractionation and an attempted integration. Brain 110, 1273-1296, 1987.

27. Warrington, E. K. and McCarthy, R. A., Multiple meaning systems in the brain-a case for visual semantics. Neuropsychologia 32, 1465-1474, 1994.

28. Warrington, E. K. and Shallice, T., Category specific semantic impairments. Brain 107, 829-854, 1984. 